\title{
Coarctação de aorta: aortoplastia com interposição da artéria subclávia esquerda (Técnica de Teles Mendonça)
}

\author{
Aortic coarctation: Aortoplasty with interposition of the left subclavian artery (the Teles Mendonça \\ Technique)
}

Ulisses Alexandre CROTI, Domingo Marcolino BRAILE, Carlos Henrique DE MARCHI, Lílian BEANI

RBCCV 44205-897

\section{DADOS CLÍNICOS}

Criança com 47 dias de vida, 3695 g, masculino, branco. Encaminhado para nosso Serviço com diagnóstico de cardiopatia congênita grave em uso de furosemida, digoxina e captopril. Desde o nascimento com cansaço às mamadas, que evoluiu para taquidispnéia, sendo internado na unidade de terapia intensiva com diagnóstico de broncopneumonia e necessitando ventilação mecânica. MEG, corado, hidratado, intubado. Tórax simétrico, ictus cordis palpável no $4^{\circ}$ espaço intercostal esquerdo. Ritmo cardíaco regular em dois tempos, bulhas normofonéticas com sopro ejetivo +/6+ em borda esternal esquerda. Ausculta pulmonar com estertores crepitantes bilateral. Abdome globoso, fígado a $4 \mathrm{~cm}$ do rebordo costal direito, ruídos hidroaéreos presentes. Membros inferiores com pulsos diminuídos, pressão arterial nos membros superiores 130/50 mmHg e membros inferiores 40/ $20 \mathrm{mmHg}$, oximetria de pulso nos membros superiores de $90 \%$.

\section{ELETROCARDIOGRAMA}

Ritmo sinusal, freqüência de $125 \mathrm{bpm}$. SÂP $+60^{\circ}$, intervalo PR 0,16 s, QTc 50s, QRS 0.08s, com sinais de alteração da repolarização do ventrículo esquerdo e sobrecarga do ventrículo direito.

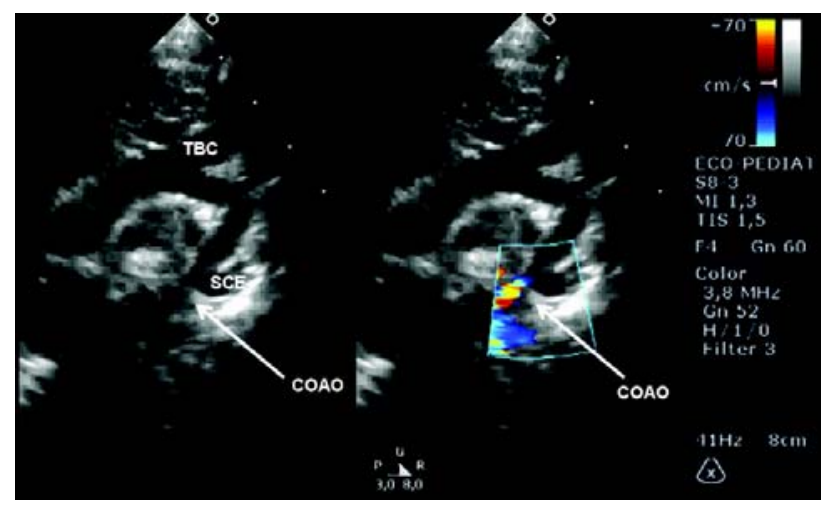

Fig. 1 - Ecocardiograma pré-operatório. COAO: coarctação de aorta, TBC: tronco braquiocefálico, SCE: artéria subclávia esquerda

\section{RADIOGRAMA}

Situs solitus visceral, índice cardiotorácico de 0,64 com proeminência das cavidades direitas. Trama vascular aumentada e cúpulas diafragmáticas livres.

Serviço de Cirurgia Cardiovascular Pediátrica de São José do Rio Preto - Hospital de Base - Faculdade de Medicina de São José do Rio Preto, SP.

Endereço para correspondência: Ulisses Alexandre Croti Hospital de Base - FAMERP - Avenida Brigadeiro Faria Lima, 5544 CEP 15090-000 - São José do Rio Preto - SP. 


\section{ECOCARDIOGRAMA}

Coarctação de aorta com importante repercussão hemodinâmica, gradiente de $80 \mathrm{mmHg}$, localizada após a emergência da artéria subclávia esquerda e com diâmetro interno de 1,7 mm, sendo a aorta 5,1 mm (Figura 1). Estenose valvar aórtica de grau discreto com pico de $29 \mathrm{mmHg}$. Forame oval pérvio. Hipertensão arterial pulmonar de grau discreto com pressão sistólica de $43 \mathrm{mmHg}$. Canal arterial fechado e presença de pequena colateral sistêmico-pulmonar da aorta descendente para a artéria pulmonar.

\section{DIAGNÓSTICO}

A história clínica e o exame físico extremamente sugestivo de coarctação de aorta foram confirmados pelo ecocardiograma, que forneceu riqueza de detalhes anatômicos. O quadro clínico e o início da falência dos órgãos orientaram para operação de urgência.

\section{OPERAÇÃO}

Toracotomia látero-posterior esquerda, dissecção extensa do arco aórtico, artéria subclávia e aorta descendente com ampla mobilização. Ligadura e secção do canal arterial (Figura 2). Secção transversal da aorta acima e abaixo da região coarctada (justaductal), secção da artéria subclávia esquerda, incisão longitudinal nas faces anterior da aorta e posterior da artéria subclávia. Anastomose término-terminal da parede posterior da aorta (Figura 3) e reconstrução final com a artéria subclávia esquerda em "telha", ampliando a região anterior na área ressecada e mantendo o fluxo sangǘneo para o membro superior esquerdo [1,2] (Figura 4). Todas as anastomoses foram realizadas com fios absorvíveis de polidioxanone 6-0 e a aorta ficou com fluxo interrompido por 37 minutos, devido à pouca experiência com a técnica, porém não ocorreram lesões neurológicas. O ecocardiograma após 45 dias demonstrou a região tratada com gradiente residual de 22 mmHg, dado que não se refletiu clinicamente e a criança está sendo acompanhada com exames periódicos, já que a técnica permite alto potencial de crescimento dos tecidos com baixa possibilidade de reestenose.

\section{REFERENNCIAS}

1. Mendonça JT, Carvalho MR, Costa RK, Franco Filho E. Coarctation of the aorta: a new surgical technique. J Thorac Cardiovasc Surg. 1985;90(3):445-7.

2. Meier MA, Lucchese FA, Jazbik W, Nesralla IA, Mendonça JT. A new technique for repair of aortic coarctation. Subclavian flap aortoplasty with preservation of arterial blood flow to the left arm. J Thorac Cardiovasc Surg. 1986;92(6):1005-12.

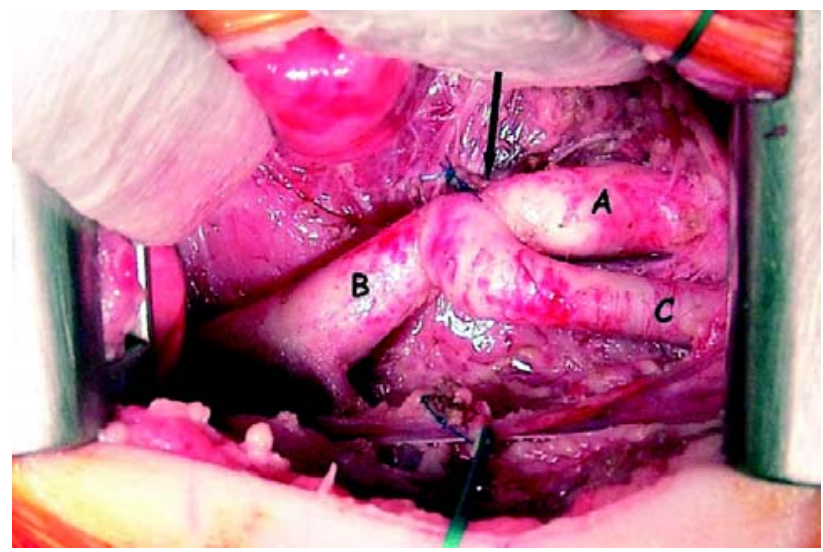

Fig. 2 - Aspecto inicial após dissecção das estruturas e ligadura do canal arterial. A região da coarctação é indicada pela seta, A: arco aórtico, B: aorta descendente, C: artéria subclávia esquerda

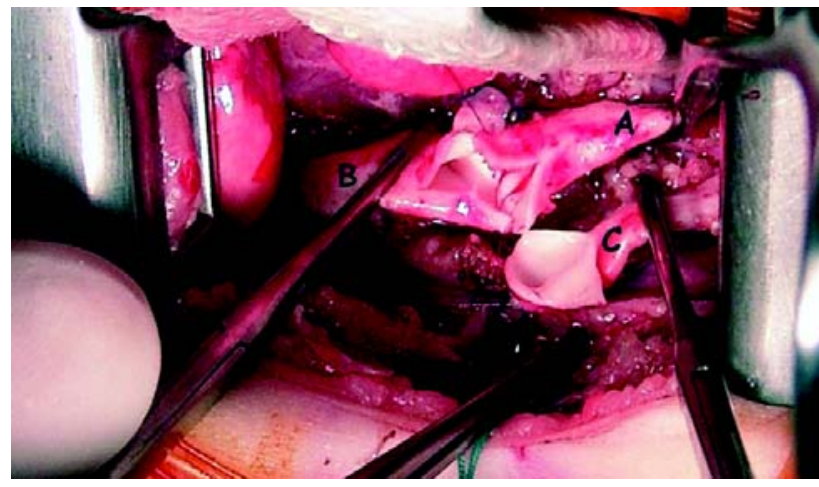

Fig. 3-Parede posterior da aorta anastomosada término-terminal. Incisão longitudinal na face anterior da aorta e posterior da artéria subclávia. A: arco aórtico, B: aorta descendente, C: artéria subclávia esquerda

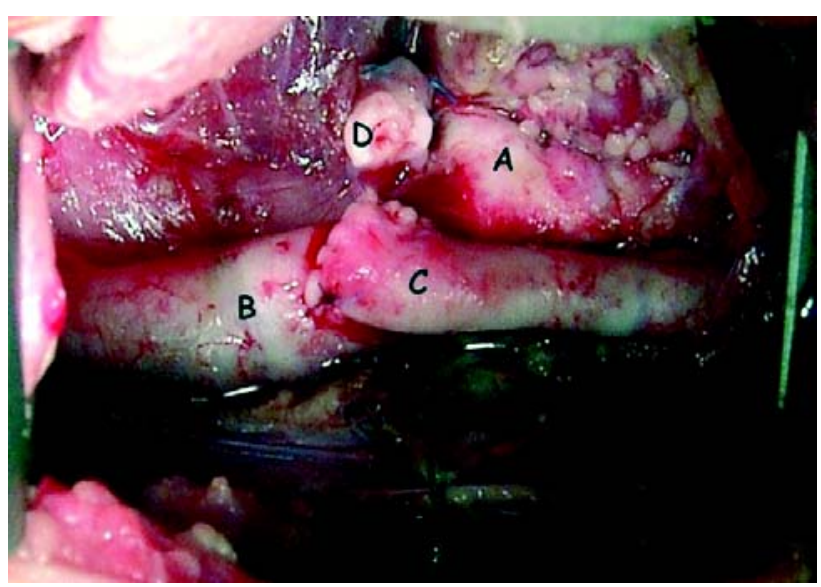

Fig. 4 - Aspecto final. A: arco aórtico, B: aorta descendente, C: artéria subclávia esquerda, $D$ : resquício da região coarctada junto ao canal arterial ligado 